\title{
Flowering responses to light and temperature
}

\author{
Li Li, Xu Li, Yawen Liu \& Hongtao Liu* \\ National Key Laboratory of Plant Molecular Genetics, Institute of Plant Physiology and Ecology, Shanghai Institutes for Biological Sciences, \\ Chinese Academy of Sciences, Shanghai 200032, China
}

Received April 28, 2015; accepted May 20, 2015; published online March 1, 2016

\begin{abstract}
Light and temperature signals are the most important environmental cues regulating plant growth and development. Plants have evolved various strategies to prepare for, and adapt to environmental changes. Plants integrate environmental cues with endogenous signals to regulate various physiological processes, including flowering time. There are at least five distinct pathways controlling flowering in the model plant Arabidopsis thaliana: the photoperiod pathway, the vernalization/thermosensory pathway, the autonomous floral initiation, the gibberellins pathway, and the age pathway. The photoperiod and temperature/vernalization pathways mainly perceive external signals from the environment, while the autonomous and age pathways transmit endogenous cues within plants. In many plant species, floral transition is precisely controlled by light signals (photoperiod) and temperature to optimize seed production in specific environments. The molecular mechanisms by which light and temperature control flowering responses have been revealed using forward and reverse genetic approaches. Here we focus on the recent advances in research on flowering responses to light and temperature.
\end{abstract}

flowering, light signaling, temperature, photoperiod, vernalization, thermosensory pathway

Citation: $\quad$ Li, L., Li, X., Liu, Y., and Liu, H. (2016). Flowering responses to light and temperature. Sci China Life Sci 59, 403-408. doi: 10.1007/s11427-015-4910-8

\section{INTRODUCTION}

The timing of the developmental transition from vegetative growth to reproductive development (flowering transition) is crucial for reproductive success in higher plants. In many plant species, flowering is controlled by the seasonal cue day length and also temperature. Here we focus on photoperiodic responses and also flowering responses to temperatures.

\section{PHOTOPERIODIC RESPONSES}

Both light quality (spectral quality) and photoperiod (day length) affect the initiation of flowering. As a facultative long day species, Arabidopsis flowers more rapidly under long-day (LD) photoperiod than under short-day (SD) con-

*Corresponding author (email: htliu@ sibs.ac.cn) dition (Andres and Coupland, 2012; Romera-Branchat et al., 2014). The $C O$ (constans) and FT (flowering locus T) genes are among the most important regulators that integrate light and temporal signals to control floral initiation in response to photoperiods (Kobayashi et al., 1999; Putterill et al., 1995). CO is a zinc finger transcription regulator that promotes flowering, at least partially, by activating the expression of FT (Onouchi et al., 2000; Samach et al., 2000). FT is a RAF kinase-related protein, which acts as a long-distance signal, migrating from leaves through the vascular system to the apical meristem (Corbesier et al., 2007; Lifschitz et al., 2006).

\section{Transcriptional and post transcriptional regulation of CO}

The circadian clock and light signals regulate the expression of $\mathrm{CO}$, while light signals stabilize the $\mathrm{CO}$ protein (Valverde et al., 2004; Yanovsky and Kay, 2002). Plants 
possess a serials of photoreceptors to absorb light at different wavelengths. There are five red/far-red light receptors phytochromes (PhyA-E) (Quail, 2002), seven blue light receptors, including two phototropins (Phot1 and Phot2) (Briggs and Christie, 2002), two cryptochromes (CRY1 and CRY2) (Lin, 2002), and three LOV/F-box/Kelch domain proteins FKF1 (flavin binding, kelch repeat, F-box protein 1), ZTL (zeitlupe), LKP2 (lov kelch protein 2) (Demarsy and Fankhauser, 2009), and one ultraviolet-B receptor UVR8 (Rizzini et al., 2011). These receptors harvest different qualities of light and work together to modulate physiological responses, such as flowering.

Light activation of $C O$ transcription is induced by the blue-light-dependent interaction between the FMN (flavin mononucleotide) containing F-box blue light photoreceptor FKF1 and the plant specific protein GI (gigantea). Both FKF and GI are also circadian clock components (Baudry et al., 2010; Sawa et al., 2007). The abundance of FKF mRNA and FKF protein show robust diurnal rhythmic with peaks in the afternoon, just coincides with the peak of CO. FKF1 was shown to simultaneously interact with the cycling dof factor 1 (CDF1) transcription factor and control the stability of CDF1, which represses the transcription of $C O$ and FT. The blue light dependent interaction of FKF1 and GI was shown to induce the degradation of the CDF1 protein and remove the repression of CDF1 on $C O$ and FT in the afternoon (Fornara et al., 2009; Imaizumi et al., 2005; Song et al., 2012).

The cryptochromes are photolyase-like blue light receptors that originally discovered in Arabidopsis, and later found widely exist in almost all species. The photoexcited CRY undergoes a serial of biophysical and biochemical changes, including circular electron transfer, phosphorylation, ubiquitination and conformational changes to alter gene expressions at both the transcriptional and posttranslational levels (Liu et al., 2010; Liu et al., 2011). CRY2 was shown to activate FT mRNA expression in response to blue light by suppressing the degradation of $\mathrm{CO}$ (Valverde et al., 2004; Yanovsky and Kay, 2002). The action of CRY2 on CO protein stability may be explained, at least partially, by the light-independent interaction between CRY2 and COP1 (constitutive photomorphogenic 1), and the blue-lightdependent interaction between CRY2 and SPA1 (suppressor of phya). COP1 is an E3 ubiquitin ligase that targets $\mathrm{CO}$ and several other transcription factors for degradation (Jang et al., 2008; Wang et al., 2001; Zuo et al., 2011), and photoexcited cryptochrome 2 interacts with SPA1 to suppress $\mathrm{COP} 1$-dependent degradation of $\mathrm{CO}$. It has been proven that COP1 physically interacts with $\mathrm{CO}$ in vivo, and that COP1 facilitates the ubiquitination of $\mathrm{CO}$ in vitro, while SPAs interact with COP1 to form a complex and target CO for degradation under dark condition (Laubinger et al., 2006; Liu et al., 2008b).

Phytochromes are synthesized in the cytosol in their inactive form (Pr), and change to their active form (pfr) by light perception, which also induces their nuclear localiza- tion to trigger light-induced responses. PhyA stabilizes $\mathrm{CO}$ by suppressing the activity of the SPAs-COP1 complex. In contrast to PhyA, PhyB delays flowering by promoting the degradation of $\mathrm{CO}$, although the molecular mechanism of phyB-mediated $\mathrm{CO}$ destabilization remains unclear (Valverde et al., 2004). A recently study found that a unique PHL (phytochrome-dependent late-flowering) gene was involved in the PhyB-dependent regulation of flowering. PHL can physically interact with PhyB and CO. Genetic and biochemical evidences suggest that PHL may suppress the activity of PhyB on CO by forming a phyB-PHL-CO tripartite complex (Endo et al., 2013). Recently, it was shown that the blue light photoreceptor FKF1 was also involved in the regulation of $\mathrm{CO}$ stability: blue light enhanced the interaction between FKF1 and CO, so that FKF1 stabilized $\mathrm{CO}$ in the afternoon in LD condition (Song et al., 2012). Besides COP1, HOS1 (high expression of osmotically responsive gene 1), a RING-finger containing E3 ubiquitin ligase also contributes to the instability of CO. HOS1 interacts with $\mathrm{CO}$ both in vitro and in planta, and HOS1 regulates the abundance of $\mathrm{CO}$, particularly during daylight and under cold temperatures (Jung et al., 2012; Lazaro et al., 2012). It seems that HOS1 integrates light and temperature signals to regulate the abundance of $\mathrm{CO}$, but it is still unclear how light and temperatures modulate the E3 ubiquitin ligase activity of HOS1.

\section{Transcriptional regulation of $F T$}

The amount of $F T$ mRNA largely determinates the flowering time. The transcriptional activation of FT in response to LD conditions is the limiting step in the photoperiodic induction of flowering in Arabidopsis. CO is the main transcriptional activator of $F T$, it binds to the proximal region of the $F T$ promoter by recognizing two $\mathrm{CO}$ responsive elements (CORE) that are required for $F T$ activation (Adrian et al., 2010). Recently, two interdependent regulatory regions in the $F T$ promoter were shown to be necessary and sufficient to convey the photoperiodic responsiveness to FT (Liu et al., 2014). It was shown that nuclear factor Y (NF-Y) bound to a CCAAT element located around $5.3 \mathrm{~kb}$ upstream of the transcription start site of FT (Cao et al., 2014), and certain NF-Y subunit paralogues were required for CO to activate FT (Kumimoto et al., 2010).

Cryptochromes, FKF1 and phytochromes may also modulate $F T$ transcription. For example, the bHLH transcription factor CIB1 is the first blue-light-dependent CRY2interacting protein. It promotes flowering in a CRY dependent manner by activating the transcription of FT. CIB1 has several homologs (CIB2, CIB4, and CIB5) that function redundantly with CIB1 to activate the transcription of FT by forming different heterodimers. All of these homologs positively regulate CRY2-mediated photoperiodic flowering, and also FT transcription (Liu et al., 2008a; Liu et al., 2013). PhyB controls floral initiation in response to the 
changes in light quality by suppressing PFT1 (phytochromes and flowering time 1), which modulates FT mRNA expression via not only a CO-dependent pathway, but also a CO-independent path-way (Cerdan and Chory, 2003). PFT1 has been shown to be a subunit (Med25) of the mediator (Backstrom et al., 2007), suggesting that PFT1 is most likely involved in the regulation of transcription. Moreover, it has also been found that the phytochrome-interacting bHLH transcription factor, PIF3, inhibits FT mRNA expression to suppress floral initiation (Oda et al., 2004), while PIF4 activates $F T$ transcription in response to increasing temperature (Kumar et al., 2012). Recently, VOZ1 (vascular plant one-zinc finger 1) and VOZ2 were identified as PhyB-interacting factors, and they promoted flowering through activating FT expression (Yasui et al., 2012). The FKF1 protein was shown to control robust FT mRNA induction by removing CDF1, transcriptional repressor of both $C O$ and FT (Song et al., 2012).

\section{Movement of the FT protein}

FT acts as a long distance signal, migrating from leaves through the vascular system to the apical meristem to induce flowering (Corbesier et al., 2007; Lifschitz et al., 2006). In the apex stem cells, FT forms protein complexes with bZIP transcription factors, such as FD to regulate transcription of floral meristem identity genes, resulting in floral initiation (Abe et al., 2005; Taoka et al., 2011; Wigge et al., 2005). Biochemical in vitro studies as well as crystallization studies have indicated that the interaction between FT and FD is indirect and is mediated by a 14-3-3 protein. 14-3-3 proteins act as intracellular receptors for florigen in shoot apical cell (Taoka et al., 2011). BRC1 (branched 1) is expressed in axillary meristems and was proposed to act as a repressor of axillary meristem differentiation (Aguilar-Martinez et al., 2007). BRC1 also delays flowering of axillary branches by interacting with FT, and the mechanism of interaction would be different from FD, given that 14-3-3 is not required for the interaction between BRC1 and FT (Niwa et al., 2013).

The FT protein is smaller than the size limit of the plasmodesmata, so it may passively move into the sieve elements via diffusion. An active transport mechanism is also possible since the abundance of FT is low. FTIP1 (ftinteracting protein 1), a FT interacting protein that is present in the plasmodesmata of the phloem companion cells in Arabidopsis was shown to be required for the transport of FT (Liu et al., 2012). FT was also shown to bind to lipids in vitro, and the capacity to bind to lipids might contribute to its movement and activity in vivo (Nakamura et al., 2014).

\section{FLOWERING RESPONSES TO TEMPERATURE}

Plants response to wide variation of environment tempera- tures with a highly accurate system for measurement: vernalization for floral promotion to meet a prolonged cold condition; delaying flowering under low ambient temperatures; while facilitating flowering in warmer conditions. Compared with light regulated flowering, less is known about how plants perceive temperature signals. However, recent advances have revealed multiple molecular mechanisms in this biological process, and some key components were identified.

\section{Flowering responses to prolonged cold temperature: vernalization}

Many studies on the molecular-mechanism of temperature effects on flowering were focused on vernalization: that is the process by which plants acquire the ability to flower after a prolonged cold treatment. Vernalization modifies flowering mainly by epigenetic silencing of FLC (flowering locus c) expression, a gene encoding a MADS box protein that represses flowering (Michaels and Amasino, 1999; Sheldon et al., 1999). FLC delays flowering by blocking the transcription of genes in the photoperiodic pathway, such as $F T$. FLC binds directly to the chromatin of FT and SOC1 to repress their transcription. Three distinct phases are involved in vernalizatin in Arabidopsis thaliana: the establishment of a vernalization requirement through activation of $F L C$; the dynamic reprogramming of FLC chromatin during the cold treatment; and the epigenetic maintenance of FLC silencing throughout development (Song et al., 2013). The activation of FLC expression requires many conserved chromatin modifiers influencing histone $\mathrm{H} 3$ lysine 4 and lysine 36 (Crevillen and Dean, 2011). Polycomb repressive complex 2 (PRC2) increases $\mathrm{H} 3 \mathrm{~K} 27 \mathrm{me} 3$ throughout the FLC locus, and is required for stable silencing FLC after vernalization. Recently, it was reported that long noncoding RNAs (lncRNAs) COOLAIR and COLDAIR were expressed at higher levels during vernalization, and were involved in regulating the FLC level (Heo and Sung, 2011; Swiezewski et al., 2009). The mechanism of age-dependent responses to vernalization was recently examined in Cardamine flexuosa and Arabidopsis alpine. In both of these species, flowering in response to vernalization requires both down-regulation of $F L C$ and reduced microRNA156 (miR156) levels. miR156 is a marker for age, and it targets the mRNAs of SPL transcription factors (Bergonzi et al., 2013; Zhou et al., 2013). HOS1, the E3 ubiquitin ligase of $\mathrm{CO}$, was also reported to be involved in regulating FLC. HOS1 was shown to promote FLC transcription through chromatin remodeling under short-term cold stress conditions by binding to FLC chromatin in a cold-dependent manner (Jung et al., 2013).

\section{Flowering responses to ambient growth temperatures}

Leaving vernalization aside, variations in ambient growth 
temperatures also dramatically affect the flowering time of plants. For example, high ambient temperatures promote flowering: under SD conditions, warm temperatures can largely substitute for LD conditions to promote flowering. Extensive genetic analyses in Arabidopsis have uncovered some mutants and ecotypes that show aberrant thermosensitivity. Blazquez and colleagues found that some lateflowering autonomous-pathway mutants such as $f c a$, fve and fy flowered at the same time regardless of the ambient temperature. This observation indicated that genes of the autonomous pathway that were previously thought to act only independently of environmental cues are centrally involved in mediating the effects of ambient temperature. Ambient temperature also controls flowering mainly through affecting the expression of $F T$ (Blazquez et al., 2003). According to current knowledge, FCA, FVE, and FY down regulate the floral repressor $F L C$. However, further analyses have shown that decreased temperature only modestly alters FLC expression, and does not suppress the effect of FCA, FVE or FY on FLC expression. These findings indicated that there was only a weak relationship between FLC and temperature sensing. flc null mutants were still responsive to changes in ambient temperatures $\left(16^{\circ} \mathrm{C}\right.$ vs. $\left.23^{\circ} \mathrm{C}\right)$, which almost rules out a role of FLC in the temperature response. Different from their action in the vernalization pathway, FCA and FVE may control flowering time in response to ambient temperatures through an FLC-independent pathway (Blazquez et al., 2003). On the other hand, the transcriptional data in the online databases show that $F L C$ affects the abundance of temperature-responsive genes between 12 and $27^{\circ} \mathrm{C}$. Experiments on the temperature compensation of the circadian clock showed that FLC lengthened the circadian period specifically at $27^{\circ} \mathrm{C}$ (Edwards et al., 2006), demonstrating a remarkable role of FLC in modulating sensitivity to higher temperature. The flowering of $f l c-3$ mutants was insensitive to higher temperature $\left(23-27^{\circ} \mathrm{C}\right)$. This finding combined with those previous research on autonomous mutants, raised the hypothesis that higher FLC levels contributed to the failure of those autonomous pathway mutants to respond to increased temperatures. The double mutants of $f l c$ with autonomous pathway mutants ( $f c a$, fve, fpa, fld, Id) showed a similar response to temperature as that of the flc single mutant, while friflc plants had similar flowering times at 23 and $16^{\circ} \mathrm{C}$ (Balasubramanian et al., 2006). Those results indicated that elevated $F L C$ levels in the autonomous pathway mutants might be responsible for their failures to respond to thermal induction of flowering. These findings support the role of $F L C$ as a mediator of the temperature-regulated flowering.

MADS-domain proteins FLM (flowering locus $\mathrm{m}$ ) and SVP (short vegetative phase) have been shown to be regulated by ambient temperature and are thought to be involved in ambient temperature regulated flowering (Balasubramanian et al., 2006; Lee et al., 2007). Recent studies have shown that ambient temperature controlled alternative splicing of FLM and the protein stability of SVP are critical for temperature regulated flowering. The levels of FLM $\beta$, an alternative splicing form of FLM, increase at lower temperatures, while the spliced form of FLM $\gamma$ is produced at higher ambient temperatures. FLM $\beta$ interacts with SVP, and then the FLM $\beta$-SVP complex binds to DNA and represses flowering. FT, TSF and SOCl are major downstream targets of SVP, FLM and FLC in ambient temperature-responsive flowering. SVP is degraded at high temperatures, reducing the abundance of the FLM $\beta$-SVP complex, which allows the plant to flower (Hwan Lee et al., 2014; Lee et al., 2013). FLM $\gamma$ and FLM $\beta$ compete for interaction with SVP, the FLM $\beta$-SVP complex predominantly forms at low temperature and prevents flowering, while the competing FLM $\gamma$-SVP complex is unable to bind to DNA and acts as a dominant negative activator of flowering at high temperatures (Pose et al., 2013).

The MYB transcription factor EFM (early flowering myb protein) was shown to play an important role in directly repressing FT expression in the leaf vasculature. EFM mediates the effects of ambient temperature on flowering and is promoted by SVP. Furthermore, EFM interacts with JMJ30, a H3K36 me2 demethylase that plays a role in the circadian clock. The interaction between EFM and JMJ30 affects the H3K36me2 dynamics of $F T$, thus EFM may be an important convergence point that mediates plant response to temperature and light to determine the flowering time (Yan et al., 2014).

PIF4, a protein involved in light signaling, was also shown to function in ambient temperature regulated floral initiation, although it was not reported to function in light quality-regulated flowering. Only under non-inductive SD conditions, PIF4 binds directly to the $F T$ locus in a temperature-dependent manner to promote $F T$ expression under higher temperatures (Kumar et al., 2012).

\section{SUMMARY}

Our understanding of the mechanisms controlling photoperiodic and temperature regulated flowering is increasing rapidly. Genes involved in these responses have been identified, and how those genes interact at the genetic level has been carried out. However, fundamental questions remain. Light signals are involved in regulating $\mathrm{CO}$ expression and protein stability, but whether light signals are also involved in regulating the transcription activity of $\mathrm{CO}$ is still unclear. Many transcription factors are involved in the transcription regulation of $F T$, some work as positive regulators, such as $\mathrm{CO}, \mathrm{CIBs}$, PIF4, GI and some work as negative regulators, such as FLC, CDFs, PIF3, TEM (Fornara et al., 2009; Imaizumi et al., 2005; Kumar et al., 2012; Liu et al., 2008a; Onouchi et al., 2000; Samach and Coupland, 2000; Sawa et al., 2007; Searle and Coupland, 2004). It remains unclear how 
these transcription factors regulate $F T$ transcription. Do they affect transcription initiation, elongation or termination? Another question is how they coordinate to regulate FT? Do they regulate $F T$ together in a complex or competitively according to different external and endogenous cues? Compared with light signal, much less is known about how plants sense and respond to temperature signals, although the histone variant H2A.Z has been proposed to mediate warm temperature signals in yeast and Arabidopsis (Kumar and Wigge, 2010). It is logical that light and temperature are associated, as daytime is normally accompanied by higher temperatures while darkness by cooler temperatures, but how do they coordinate to regulate plant development, such as flowering? Further study is required to shed light on these intriguing questions to gain a comprehensive understanding of flowering in response to changes in the environment.

This work was supported by the National Natural Science Foundation of China (31322006, 31270285), and the Hundred Talents Program of the Chinese Academy of Sciences.

Abe, M., Kobayashi, Y., Yamamoto, S., Daimon, Y., Yamaguchi, A., Ikeda, Y., Ichinoki, H., Notaguchi, M., Goto, K., and Araki, T. (2005). FD, a bZIP protein mediating signals from the floral pathway integrator FT at the shoot apex. Science 309, 1052-1056.

Adrian, J., Farrona, S., Reimer, J.J., Albani, M.C., Coupland, G., and Turck, F. (2010). cis-Regulatory elements and chromatin state coordinately control temporal and spatial expression of FLOWERING LOCUS T in Arabidopsis. Plant Cell 22, 1425-1440.

Aguilar-Martinez, J.A., Poza-Carrion, C., and Cubas, P. (2007). Arabidopsis BRANCHED1 acts as an integrator of branching signals within axillary buds. Plant Cell 19, 458-472.

Andres, F., and Coupland, G. (2012). The genetic basis of flowering responses to seasonal cues. Nat Rev Genet 13, 627-639.

Backstrom, S., Elfving, N., Nilsson, R., Wingsle, G., and Bjorklund, S. (2007). Purification of a plant mediator from Arabidopsis thaliana identifies PFT1 as the Med25 subunit. Mol Cell 26, 717-729.

Balasubramanian, S., Sureshkumar, S., Lempe, J., and Weigel, D. (2006). Potent induction of Arabidopsis thaliana flowering by elevated growth temperature. PLoS Genet 2, e106.

Baudry, A., Ito, S., Song, Y.H., Strait, A.A., Kiba, T., Lu, S., Henriques, R., Pruneda-Paz, J.L., Chua, N.H., Tobin, E.M., Kay, S.A., and Imaizumi, T. (2010). F-box proteins FKF1 and LKP2 act in concert with ZEITLUPE to control Arabidopsis clock progression. Plant Cell 22, 606-622.

Bergonzi, S., Albani, M.C., Ver Loren van Themaat, E., Nordstrom, K.J., Wang, R., Schneeberger, K., Moerland, P.D., and Coupland, G. (2013). Mechanisms of age-dependent response to winter temperature in perennial flowering of Arabis alpina. Science 340, 1094-1097.

Blazquez, M.A., Ahn, J.H., and Weigel, D. (2003). A thermosensory pathway controlling flowering time in Arabidopsis thaliana. Nat Genet 33, 168-171.

Briggs, W.R., and Christie, J.M. (2002). Phototropins 1 and 2: versatile plant blue-light receptors. Trends Plant Sci 7, 204-210.

Cao, S., Kumimoto, R.W., Gnesutta, N., Calogero, A.M., Mantovani, R., and Holt, B.F., 3rd (2014). A distal CCAAT/NUCLEAR FACTOR Y complex promotes chromatin looping at the FLOWERING LOCUS T promoter and regulates the timing of flowering in Arabidopsis. Plant Cell 26, 1009-1017.

Cerdan, P.D., and Chory, J. (2003). Regulation of flowering time by light quality. Nature 423, 881-885.

Corbesier, L., Vincent, C., Jang, S., Fornara, F., Fan, Q., Searle, I., Gia- kountis, A., Farrona, S., Gissot, L., Turnbull, C., and Coupland, G. (2007). FT protein movement contributes to long-distance signaling in floral induction of Arabidopsis. Science 316, 1030-1033.

Crevillen, P., and Dean, C. (2011). Regulation of the floral repressor gene FLC: the complexity of transcription in a chromatin context. Curr Opin Plant Biol 14, 38-44.

Demarsy, E., and Fankhauser, C. (2009). Higher plants use LOV to perceive blue light. Curr Opin Plant Biol 12, 69-74.

Edwards, K.D., Anderson, P.E., Hall, A., Salathia, N.S., Locke, J.C., Lynn, J.R., Straume, M., Smith, J.Q., and Millar, A.J. (2006). FLOWERING LOCUS $\mathrm{C}$ mediates natural variation in the hightemperature response of the Arabidopsis circadian clock. Plant Cell 18, 639-650.

Endo, M., Tanigawa, Y., Murakami, T., Araki, T., and Nagatani, A. (2013). PHYTOCHROME-DEPENDENT LATE-FLOWERING accelerates flowering through physical interactions with phytochrome B and CONSTANS. Proc Natl Acad Sci USA 110, 18017-18022.

Fornara, F., Panigrahi, K.C., Gissot, L., Sauerbrunn, N., Ruhl, M., Jarillo, J.A., and Coupland, G. (2009). Arabidopsis DOF transcription factors act redundantly to reduce CONSTANS expression and are essential for a photoperiodic flowering response. Dev Cell 17, 75-86.

Heo, J.B., and Sung, S. (2011). Vernalization-mediated epigenetic silencing by a long intronic noncoding RNA. Science 331, 76-79.

Hwan Lee, J., Sook Chung, K., Kim, S.K., and Ahn, J.H. (2014). Post-translational regulation of SHORT VEGETATIVE PHASE as a major mechanism for thermoregulation of flowering. Plant Signal Behav 9, e28193.

Imaizumi, T., Schultz, T.F., Harmon, F.G., Ho, L.A., and Kay, S.A. (2005). FKF1 F-box protein mediates cyclic degradation of a repressor of CONSTANS in Arabidopsis. Science 309, 293-297.

Jang, S., Marchal, V., Panigrahi, K.C., Wenkel, S., Soppe, W., Deng, X.W., Valverde, F., and Coupland, G. (2008). Arabidopsis COP1 shapes the temporal pattern of $\mathrm{CO}$ accumulation conferring a photoperiodic flowering response. EMBO J 27, 1277-1288.

Jung, J.H., Park, J.H., Lee, S., To, T.K., Kim, J.M., Seki, M., and Park, C.M. (2013). The cold signaling attenuator HIGH EXPRESSION OF OSMOTICALLY RESPONSIVE GENE1 activates FLOWERING LOCUS $\mathrm{C}$ transcription via chromatin remodeling under short-term cold stress in Arabidopsis. Plant Cell 25, 4378-4390.

Jung, J.H., Seo, P.J., and Park, C.M. (2012). The E3 ubiquitin ligase HOS1 regulates Arabidopsis flowering by mediating CONSTANS degradation under cold stress. J Biol Chem 287, 43277-43287.

Kobayashi, Y., Kaya, H., Goto, K., Iwabuchi, M., and Araki, T. (1999). A pair of related genes with antagonistic roles in mediating flowering signals. Science 286, 1960-1962.

Kumar, S.V., Lucyshyn, D., Jaeger, K.E., Alos, E., Alvey, E., Harberd, N.P., and Wigge, P.A. (2012). Transcription factor PIF4 controls the thermosensory activation of flowering. Nature 484, 242-245.

Kumar, S.V., and Wigge, P.A. (2010). H2A.Z-containing nucleosomes mediate the thermosensory response in Arabidopsis. Cell 140, $136-147$.

Kumimoto, R.W., Zhang, Y., Siefers, N., and Holt, B.F., 3rd (2010). NF-YC3, NF-YC4 and NF-YC9 are required for CONSTANSmediated, photoperiod-dependent flowering in Arabidopsis thaliana. Plant J 63, 379-391.

Laubinger, S., Marchal, V., Le Gourrierec, J., Wenkel, S., Adrian, J., Jang, S., Kulajta, C., Braun, H., Coupland, G., and Hoecker, U. (2006). Arabidopsis SPA proteins regulate photoperiodic flowering and interact with the floral inducer CONSTANS to regulate its stability. Development 133, 3213-3222.

Lazaro, A., Valverde, F., Pineiro, M., and Jarillo, J.A. (2012). The Arabidopsis E3 ubiquitin ligase HOS1 negatively regulates CONSTANS abundance in the photoperiodic control of flowering. Plant Cell 24, 982-999.

Lee, J.H., Ryu, H.S., Chung, K.S., Pose, D., Kim, S., Schmid, M., and Ahn, J.H. (2013). Regulation of temperature-responsive flowering by MADS-box transcription factor repressors. Science 342, 628-632.

Lee, J.H., Yoo, S.J., Park, S.H., Hwang, I., Lee, J.S., and Ahn, J.H. (2007). Role of SVP in the control of flowering time by ambient tem- 
perature in Arabidopsis. Genes Dev 21, 397-402.

Lifschitz, E., Eviatar, T., Rozman, A., Shalit, A., Goldshmidt, A., Amsellem, Z., Alvarez, J.P., and Eshed, Y. (2006). The tomato FT ortholog triggers systemic signals that regulate growth and flowering and substitute for diverse environmental stimuli. Proc Natl Acad Sci USA 103, 6398-6403.

Lin, C. (2002). Blue light receptors and signal transduction. Plant Cell 2002S, S207-S225.

Liu, B., Liu, H., Zhong, D., and Lin, C. (2010). Searching for a photocycle of the cryptochrome photoreceptors. Curr Opin Plant Biol 13, $578-586$.

Liu, H., Liu, B., Zhao, C., Pepper, M., and Lin, C. (2011). The action mechanisms of plant cryptochromes. Trends Plant Sci 16, 684-691.

Liu, H., Yu, X., Li, K., Klejnot, J., Yang, H., Lisiero, D., and Lin, C. (2008a). Photoexcited CRY2 interacts with CIB1 to regulate transcription and floral initiation in Arabidopsis. Science 322, 1535-1539.

Liu, L., Adrian, J., Pankin, A., Hu, J., Dong, X., von Korff, M., and Turck, F. (2014). Induced and natural variation of promoter length modulates the photoperiodic response of FLOWERING LOCUS T. Nat Commun 5, 4558.

Liu, L., Liu, C., Hou, X., Xi, W., Shen, L., Tao, Z., Wang, Y., and Yu, H. (2012). FTIP1 is an essential regulator required for florigen transport. PLoS Biol 10, e1001313.

Liu, L., Zhang, Y., Li, Q., Sang, Y., Mao, J., Lian, H., Wang, L., and Yang, H. (2008b). COP1-mediated ubiquitination of CONSTANS is implicated in cryptochrome regulation of flowering in Arabidopsis. Plant Cell 20, 292-306.

Liu, Y., Li, X., Li, K., Liu, H., and Lin, C. (2013). Multiple bHLH proteins form heterodimers to mediate CRY2-dependent regulation of flowering-time in Arabidopsis. PLoS Genet 9, e1003861.

Michaels, S.D., and Amasino, R.M. (1999). FLOWERING LOCUS C encodes a novel MADS domain protein that acts as a repressor of flowering. Plant Cell 11, 949-956.

Nakamura, Y., Andres, F., Kanehara, K., Liu, Y.C., Dormann, P., and Coupland, G. (2014). Arabidopsis florigen FT binds to diurnally oscillating phospholipids that accelerate flowering. Nat Commun 5, 3553 .

Niwa, M., Daimon, Y., Kurotani, K., Higo, A., Pruneda-Paz, J.L., Breton, G., Mitsuda, N., Kay, S.A., Ohme-Takagi, M., Endo, M., and Araki, T. (2013). BRANCHED1 interacts with FLOWERING LOCUS $\mathrm{T}$ to repress the floral transition of the axillary meristems in Arabidopsis. Plant Cell 25, 1228-1242.

Oda, A., Fujiwara, S., Kamada, H., Coupland, G., and Mizoguchi, T. (2004). Antisense suppression of the Arabidopsis PIF3 gene does not affect circadian rhythms but causes early flowering and increases FT expression. FEBS Lett 557, 259-264.

Onouchi, H., Igeno, M.I., Perilleux, C., Graves, K., and Coupland, G. (2000). Mutagenesis of plants overexpressing CONSTANS demonstrates novel interactions among Arabidopsis flowering-time genes. Plant Cell 12, 885-900.

Pose, D., Verhage, L., Ott, F., Yant, L., Mathieu, J., Angenent, G.C., Immink, R.G., and Schmid, M. (2013). Temperature-dependent regulation of flowering by antagonistic FLM variants. Nature 503, 414-417.

Putterill, J., Robson, F., Lee, K., Simon, R., and Coupland, G. (1995). The CONSTANS gene of Arabidopsis promotes flowering and encodes a protein showing similarities to zinc finger transcription factors. Cell 80, 847-857.

Quail, P.H. (2002). Photosensory perception and signalling in plant cells: new paradigms? Curr Opin Cell Biol 14, 180-188.

Rizzini, L., Favory, J.J., Cloix, C., Faggionato, D., O’Hara, A., Kaiserli,
E., Baumeister, R., Schafer, E., Nagy, F., Jenkins, G.I., and Ulm, R. (2011). Perception of UV-B by the Arabidopsis UVR8 protein. Science 332, 103-106.

Romera-Branchat, M., Andres, F., and Coupland, G. (2014). Flowering responses to seasonal cues: what's new? Curr Opin Plant Biol 21C, 120-127.

Samach, A., and Coupland, G. (2000). Time measurement and the control of flowering in plants. BioEssays: news and reviews in molecular. Cell Dev Biol 22, 38-47.

Samach, A., Onouchi, H., Gold, S.E., Ditta, G.S., Schwarz-Sommer, Z., Yanofsky, M.F., and Coupland, G. (2000). Distinct roles of CONSTANS target genes in reproductive development of Arabidopsis. Science 288, 1613-1616.

Sawa, M., Nusinow, D.A., Kay, S.A., and Imaizumi, T. (2007). FKF1 and GIGANTEA complex formation is required for day-length measurement in Arabidopsis. Science 318, 261-265.

Searle, I., and Coupland, G. (2004). Induction of flowering by seasonal changes in photoperiod. EMBO J 23, 1217-1222.

Sheldon, C.C., Burn, J.E., Perez, P.P., Metzger, J., Edwards, J.A., Peacock, W.J., and Dennis, E.S. (1999). The FLF MADS box gene: a repressor of flowering in Arabidopsis regulated by vernalization and methylation. Plant Cell 11, 445-458.

Song, J., Irwin, J., and Dean, C. (2013). Remembering the prolonged cold of winter. Curr Biol 23, R807-R811.

Song, Y.H., Smith, R.W., To, B.J., Millar, A.J., and Imaizumi, T. (2012). FKF1 conveys timing information for CONSTANS stabilization in photoperiodic flowering. Science 336, 1045-1049.

Swiezewski, S., Liu, F., Magusin, A., and Dean, C. (2009). Cold-induced silencing by long antisense transcripts of an Arabidopsis Polycomb target. Nature 462, 799-802.

Taoka, K., Ohki, I., Tsuji, H., Furuita, K., Hayashi, K., Yanase, T., Yamaguchi, M., Nakashima, C., Purwestri, Y.A., Tamaki, S., Ogaki, Y., Shimada, C., Nakagawa, A., Kojima, C., and Shimamoto, K. (2011). 14-3-3 proteins act as intracellular receptors for rice Hd3a florigen. Nature 476, 332-335.

Valverde, F., Mouradov, A., Soppe, W., Ravenscroft, D., Samach, A., and Coupland, G. (2004). Photoreceptor regulation of CONSTANS protein in photoperiodic flowering. Science 303, 1003-1006.

Wang, H., Ma, L., Li, J., Zhao, H., and Deng, X. (2001). Direct interaction of Arabidopsis cryptochromes with COP1 in light control development. Science 294, 154-158.

Wigge, P.A., Kim, M.C., Jaeger, K.E., Busch, W., Schmid, M., Lohmann, J.U., and Weigel, D. (2005). Integration of spatial and temporal information during floral induction in Arabidopsis. Science 309, 1056-1059.

Yan, Y., Shen, L., Chen, Y., Bao, S., Thong, Z., and Yu, H. (2014). A MYB-domain protein EFM mediates flowering responses to environmental cues in Arabidopsis. Dev Cell 30: 437-448.

Yanovsky, M.J., and Kay, S.A. (2002). Molecular basis of seasonal time measurement in Arabidopsis. Nature 419, 308-312.

Yasui, Y., Mukougawa, K., Uemoto, M., Yokofuji, A., Suzuri, R., Nishitani, A., and Kohchi, T. (2012). The phytochrome-interacting vascular plant one-zinc finger1 and VOZ2 redundantly regulate flowering in Arabidopsis. Plant Cell 24, 3248-3263.

Zhou, C., Zhang, T., Wang, X., Yu, S., Lian, H., Tang, H., Feng, Z., Zozomova-Lihova, J., and Wang, J. (2013). Molecular basis of age-dependent vernalization in Cardamine flexuosa. Science 340, 1097-1100.

Zuo, Z., Liu, H., Liu, B., Liu, X., and Lin, C. (2011). Blue light-dependent interaction of CRY2 with SPA1 regulates COP1 activity and floral initiation in Arabidopsis. Curr Biol 21, 841-847.

Open Access This article is distributed under the terms of the Creative Commons Attribution License which permits any use, distribution, and reproduction in any medium, provided the original author(s) and source are credited. 